\title{
COMPARISON OF RADIATION-INDUCED TOXICITIES, TREATMENT FEASIBILITY IN CONVENTIONAL VERSUS HYPO-FRACTIONATED PROTOCOLS OF POST MASTECTOMY RADIOTHERAPY
}

\author{
Sanjal Kumar V1, Baskar P2, Sunderesan C ${ }^{3}$, Harish Kumar P. R. ${ }^{4}$ \\ 1Assistant Professor, Department of Radiotherapy, Madras Medical College, Chennai. \\ ${ }^{2}$ Associate Professor, Department of Radiotherapy, Madras Medical College, Chennai. \\ ${ }^{3}$ Assistant Professor, Department of Radiotherapy, Madras Medical College, Chennai. \\ ${ }_{4}^{4}$ Postgraduate Student, Department of Radiotherapy, Madras Medical College, Chennai.
}

ABSTRACT

\section{BACKGROUND}

Breast cancer is the most common cancer in women worldwide and has overtaken cervical cancer to become the leading cause of cancer-related mortality among women living in metropolitan cities in India with an incidence at 30 to 33 per 1,00,000 women.

Objectives- To evaluate conventional versus hypo-fractionated post mastectomy radiotherapy through a non-randomised double arm prospective study by comparing pulmonary, cardiac, dermatological, haematological and lymphoedema toxicities, treatment feasibility and local control rates.

\section{MATERIALS AND METHODS}

60 breast cancer patients who were recruited from January 2011 to April 2011 were allocated to conventional and hypofractionated arm based on outpatient registration order. All the patients were treated with Cobalt-60 teletherapy machine with Computer tomography simulation with conventional portals. Conventional arm 2 Gy per fraction for 25 fractions over 6 weeks and Hypo-fractionated 2.70 Gy for 15 fractions over 3 weeks. Patients underwent pre-treatment evaluation and during treatment and post treatment evaluation at regular intervals

\section{RESULTS}

All the toxicities were graded and compared and evaluated using SPSS software and P values were found to be statistically insignificant. Hypo-fractionated schedule was completed by all patients without any interruption; however, conventional arm treatment had to be interrupted in 3 patients.

\section{CONCLUSION}

The treatment schedule and the technique are feasible to deliver without affecting the quality of life of the patient. All the toxicities parameters are within acceptable limits. Hypo-fractionation can be easily followed in Institutions with high work load due to equal outcome.

\section{KEYWORDS}

Hypo-fractionated, PMRT, Breast Radiotherapy.

HOW TO CITE THIS ARTICLE: Kumar SV, Baskar P, Sunderesan C, et al. Comparison of radiation-induced toxicities, treatment feasibility in conventional versus hypo-fractionated protocols of post mastectomy radiotherapy. J. Evolution Med. Dent. Sci. 2018;7(06):767-770, DOI: 10.14260/jemds/2018/174

\section{BACKGROUND \\ Breast cancer is the most common cancer in women} worldwide and has overtaken cervical cancer to become the leading cause of cancer-related mortality among women living in metropolitan cities in India, with an incidence at 30 to 33 per $1,00,000$ women.(1) The rationale for postmastectomy radiation is the prevention of a local-regional recurrence. Theoretically, clinically occult persistent disease in the operative site or regional nodes could act as a source of distant metastases and eradication of this could result in an improvement in disease-free and overall survival.

'Financial or Other Competing Interest': None.

Submission 25-10-2017, Peer Review 20-01-2018,

Acceptance 27-01-2018, Published 05-02-2018.

Corresponding Author:

Sanjal Kumar V,

OP-116, Department of Radiotherapy,

Rajiv Gandhi Government General Hospital,

EVR Salai,

Parktown, Chennai.

E-mail: sanjal.bmc@gmail.com

DOI: $10.14260 /$ jemds $/ 2018 / 174$

\section{(c) (1) $(9)$}

Hypo-fractionation has been studied for a long time and different schedules have been developed to minimise the burden on machines and operators. Due to the encouraging data it has been tried quite extensively in breast cancer and a lot of data is now available showing very good local control, acceptable toxicity and equivalent cosmetic outcome.(2),(3),(4),(5)
Aims and Objectives
To evaluate conventional versus hypo-fractionated post mastectomy radiotherapy through a Non-randomised controlled double arm prospective study by comparing-
1. Pulmonary, cardiac, dermatological, haematological, toxicities and lymphoedema.
2. Feasibility option.
3. Local control.

\section{MATERIALS AND METHODS}

Study Design: Non-Randomised, controlled, double arm prospective study. Patients were recruited between January 2011 to April 2011 were allotted into the conventional and hypo-fractionated arm consecutively as per outpatient registration order. 


\section{Inclusion Criteria}

1. Post-menopausal females $>45$ years.

2. T2 with nodal involvement.

3. T3, T4 Nx, N0 to N3.

4. Post MRM with negative margins.

5. Post chemotherapy.

\section{Exclusion Criteria}

1. Breast conservation.

2. Positive Margins.

3. Metastatic disease.

4. Baseline left ventricular ejection fraction $<55 \%$.

5. Restrictive lung disease.

\section{Pre-Treatment Evaluation}

1. Clinical examination including arm circumference.

2. Metastatic workup.

3. Pulmonary function test and x-ray chest.

4. Echocardiogram and 2D Echo.

5. Complete blood count.

\begin{tabular}{|c|c|c|}
\hline & ARM A & ARM B \\
\hline Dose per fraction & $270 \mathrm{cGY}$ & $200 \mathrm{cGY}$ \\
\hline Number of fractions & 15 & 25 \\
\hline Total Dose & $\mathbf{4 0 . 5}$ & $\mathbf{5 0}$ \\
\hline Duration in weeks & 3 weeks & 6 weeks \\
\hline \multicolumn{2}{|c|}{ TreatmentExecution } \\
\hline
\end{tabular}

All patients after selection underwent conventional planning positioned supinely with the ipsilateral arm being abducted above head was turned to the opposite side. The fields are chest wall field and supraclavicular field. The target volume of chest wall field was the whole chest wall within the field and a part of lung beneath that chest wall treated in two tangential portals.

The target volume of supraclavicular field includes ipsilateral supraclavicular nodes, infraclavicular nodes and axillary nodes treated with single anterior-posterior portal.

The chest wall thickness was measured using high frequency ultrasound and it was plotted in the contour. The central lung distance was maintained below $2 \mathrm{~cm}$ and then 2D planning was done.

For the supraclavicular field, the dose was prescribed at D-max level and the remaining depth dose at mid-axillary level was calculated and a posterior axillary boost was given.

Treatment was executed using gamma rays from Co-60 Theratron phoenix.

\section{Assessment}

1. 1 week after initiating treatment- skin toxicity.

2. 2 weeks after initiating treatment- skin toxicity, haematological toxicity.

3. At the end of completion of Radiotherapy- skin toxicity, haematological toxicity, lymphoedema and chest x-ray.

4. At 2 months- skin toxicity, haematological toxicity, lymphoedema, echocardiography and pulmonary function test.

5. At 6 months- lymphoedema, echocardiography, pulmonary function test and x-ray.

\section{Statistical Analysis}

Data collected were entered in Excel Sheet and analysed using SPSS version 16.0. Two-sided independent samples ' $\mathrm{t}$ ' test was used to compare means across dichotomous variables and One-Way ANOVA test to compare means across multilevel variables. Simple calculations like percentages, proportions and mean values were derived, A type 1 error of 0.05 was considered in all analyses.

\section{RESULTS}

Patient Data- Age Distribution.

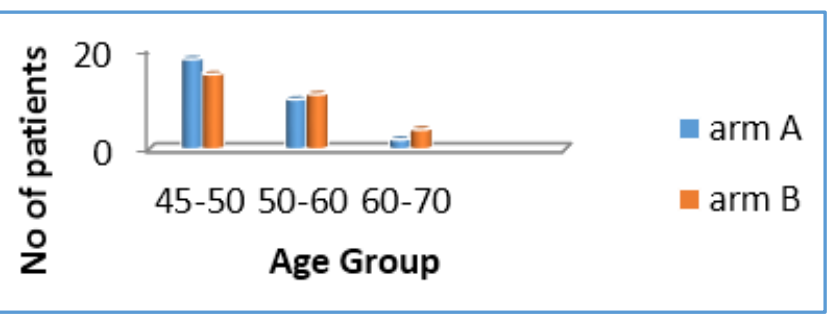

Majority of patients, 18 in hypo-fractionated arm and 15 in the conventional arm were in the 45 to 50 age group followed by 50 to 60 and 60 to 70 age group. These distributions of cases are in accordance with rest of the country age-adjusted incidence rates.

\begin{tabular}{|c|c|c|c|}
\hline \multicolumn{2}{|c|}{} & Arm A & Arm B \\
\hline $\mathbf{N}$ & Valid & $\mathbf{3 0}$ & $\mathbf{3 0}$ \\
\hline Mean & 50.8333 & 51.7000 \\
\hline Median & 49.5000 & 50.0000 \\
\hline Range & 20.00 & 20.00 \\
\hline Minimum & 45.00 & 45.00 \\
\hline \multicolumn{3}{|c|}{ Statistics } \\
\hline \multicolumn{2}{|c|}{} \\
\hline \multicolumn{2}{|c|}{} \\
\hline
\end{tabular}

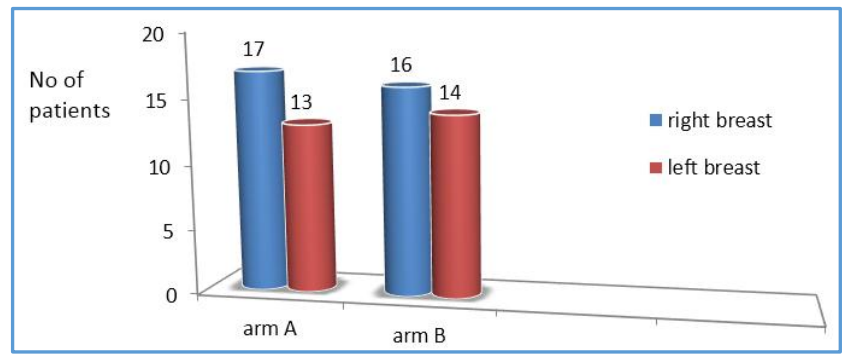

Stratification according to
Left or Right Breast

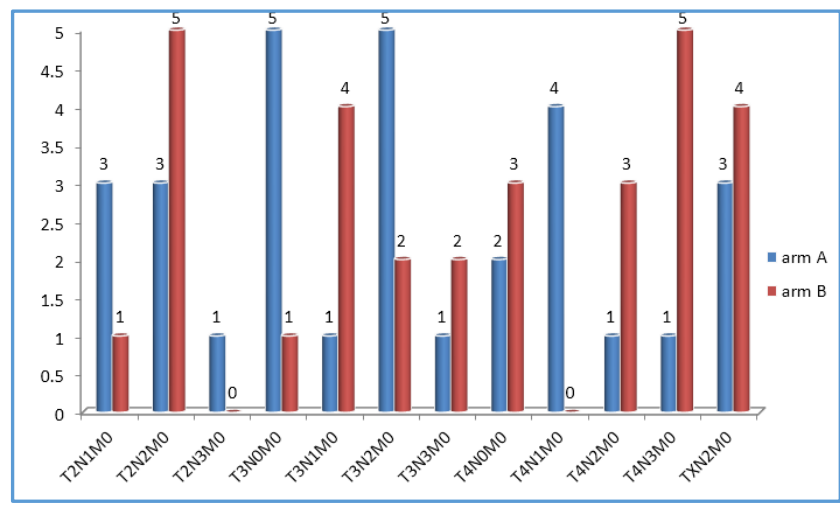

Distribution according

to AJCC Staging 


\section{All Patients were in ECOG Status 1}

Histopathological distribution showed that most of our patients were Infiltrating Ductal Carcinoma- NOS type- 88\%; 5 patients in hypo-fractionated arm were triple negative compared to 3 in conventional arm; 4 patients of the hypofractionated arm had diabetes and 5 in conventional arm; 4 patients in hypo-fractionated arm and 7 in conventional arm who were treated as inpatients and rest were all treated on an outpatient basis.

\begin{tabular}{|c|c|c|c|c|}
\hline & \multicolumn{2}{|c|}{ ARM-A } & \multicolumn{2}{|c|}{ ARM-B } \\
\hline \multirow[t]{2}{*}{ Skin toxicities } & G1-33\% & G3-0\% & G1-30 \% & G3- $0 \%$ \\
\hline & G2-23.3\% & G4-0\% & G2-20\% & G4- 0\% \\
\hline \multirow[t]{2}{*}{$\begin{array}{l}\text { Haematological } \\
\text { toxicity }\end{array}$} & \begin{tabular}{|c|} 
Mild- \\
$26.6 \%$
\end{tabular} & Severe-0\% & $\begin{array}{c}\text { Mild- } \\
23.3 \%\end{array}$ & $\begin{array}{c}\text { Severe- } \\
0 \%\end{array}$ \\
\hline & \begin{tabular}{|c|} 
Moderate- \\
$6.6 \%$ \\
\end{tabular} & & $\begin{array}{c}\text { Moderate- } \\
3.34 \% \\
\end{array}$ & \\
\hline Decrease in PFT & \multicolumn{2}{|c|}{ Nil observed } & \multicolumn{2}{|c|}{ Nil observed } \\
\hline $\begin{array}{c}\text { Radiation-induced } \\
\text { pneumonitis }\end{array}$ & \multicolumn{2}{|c|}{ Nil observed } & \multicolumn{2}{|c|}{ Nil observed } \\
\hline $\begin{array}{c}\text { Decrease in LVEF } \\
>10 \%\end{array}$ & \multicolumn{2}{|c|}{ Nil observed } & \multicolumn{2}{|c|}{ Nil observed } \\
\hline \multirow[t]{2}{*}{ Lymph-oedema } & G1-16.6\% & G3-0\% & G1-10\% & G3-0\% \\
\hline & G2-0\% & & G2-0\% & \\
\hline \multicolumn{5}{|c|}{ Toxicities Observed } \\
\hline
\end{tabular}

All the test values were analysed using SPSS software and none were found to be statistically significant.

\section{Feasibility}

Hypo-fractionated schedule was completed by all patients without any interruption. In conventional arm, treatment had to be interrupted in 3 patients for their personnel reasons. None of the early reactions during radiotherapy tend to have interrupted the treatment. The treatment schedule and the technique are feasible to deliver without affecting the quality of life of the patient.

\section{Tolerability}

All of the patients completed the schedule comfortably. Three patients had pain in the chest wall field, but they completed the treatment without delay. The treatment procedure is well tolerated by all $(100 \%)$ patients.

\section{Acute Skin Reactions}

Grade 1 toxicity noted in G1-33.4\% and G2- $23.3 \%$ in hypofractionated arm. In conventional arm it was G1-30\% and G2- 20.1\%. P value at 2 weeks, 4 weeks, 2 months and 6 months were $.038, .125, .000, .000$ and .000 . There were no G3 or G4 skin toxicities in both arms.

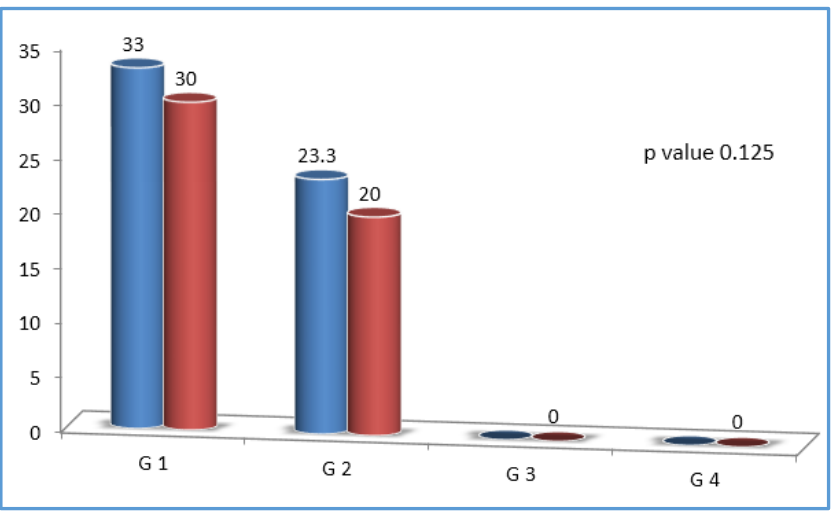

\section{Acute Chest Wall Pain}

Tenderness was noted in $10 \%$ and was tolerable and did not affect their quality of life. Most of the tenderness observed in the matching region of flap and the drainage field and in the axillary folds.

\section{Haematological Toxicities}

Mild 24\% and moderate $6.6 \%$ toxicities were observed in hypo-fractionated arm, and in conventional arm it was 23.3 and moderate $3.3 \%$. There were no severe haematological toxicities in both the arms. All patients were managed conservatively, and none needed blood transfusion. Statistically, the values between arms were insignificant.

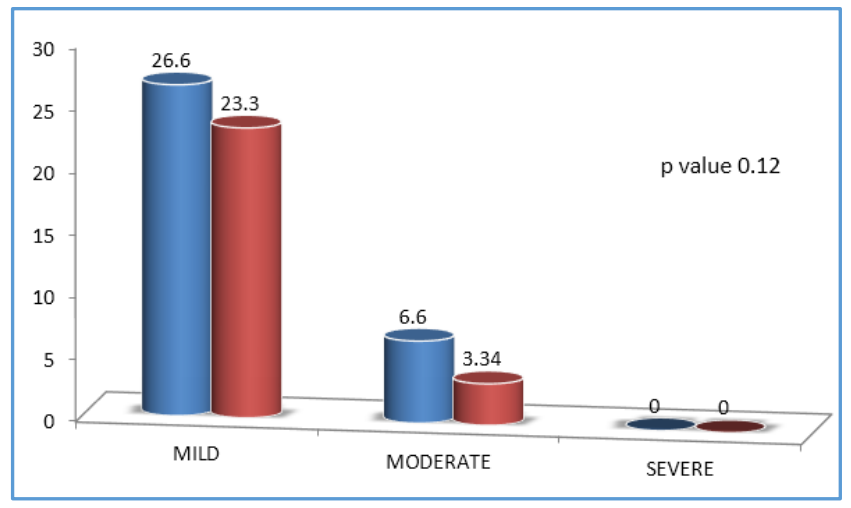

Radiation-Induced Pneumonitis

None of the patients in both the arms developed Pneumonitis.

\section{Pulmonary Function Test}

With the emphasis on FEV1 and FVC before starting RT and 2 months and 6 months, none of the patients showed a significant decrease. Statistically, the values between both arms were insignificant.

\section{Cardiac Toxicity}

The patients who had a baseline EF of $<55 \%$ were not included in the trial and subsequent follow-up studies were done at completion of RT and 2 months and 6 months, and none of the patients had a decrease in ejection fraction greater than $10 \%$, again statistically proven to be insignificant. There was no change in the ECG either. There was no significant change between left-sided and right-sided breast or cardiac parameters since majority of cardiac toxicity is expected as a late complication. There was no acute cardiac toxicities in this short follow-up in both the arms. The corresponding ' $p$ ' values before RT, completion of radiotherapy and at 6 months $.681, .405$ and .479 (CI- 1.365 to 1.525$)$.

\section{Lymphoedema}

This was taken as a clinical finding and measurement using a flexible tape was taken $20 \mathrm{cms}$ above and below the olecranon process and was taken before starting RT, completion of RT, 2 months and 6 months after RT. The observed values were G1- 16\% in hypo-fractionated arm and $13 \%$ in conventional arm. There were no grade 2 or 3 changes. 


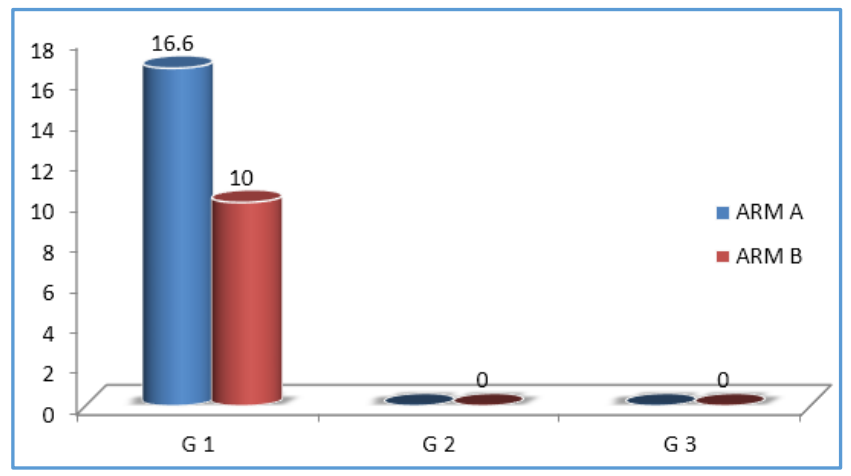

\section{DISCUSSION}

Shorter radiation schedules based on radiobiological models offer the promise of equivalent local control to standard radiation therapy by giving larger doses per fraction in shorter period.(6),(7)

The linear-quadratic model is typically used to calculate the biologically equivalent dose taking into account a larger dose per fraction over a shorter period of time.(8),(9)

This study is based on an earlier study done in Institute of Nuclear Medicine and Oncology (INMOL) using a three arm hypo-fractionated protocols in post mastectomy carcinoma breast in terms of local control, toxicity and work load. There are many single institutional trials published about hypofractionation, which have proven it to achieve equal toxicities and local control.(10)

In a retrospective study of 1624 patients, Lingos et al reported radiation pneumonitis in $1 \%$ of patients after surgery and radiation. The frequency was about $9 \%$ when three fields plus concurrent chemo was administered compared to only when two fields and sequential chemoradiation was used.

Plataniotis GA et al evaluated radiation pneumonitis in hypo-fractionation setting ( $42.5 \mathrm{~Gy} / 16 \mathrm{Fr}$ ) by HRCT in early breast cancer patients, and reported minimal and minor effects on the underlying lung parenchyma.

Lymphoedema is an established complication of both axillary lymph node dissection (ALND) and axillary radiotherapy. As is evident from our results that almost $2 / 3^{\text {rd }}$ patients never developed this problem.

Chua B reported $9.5 \%$ arm oedema with axillary dissection, $6.1 \%$ with radiation and $31 \%$ when the two modalities were combined $\mathrm{p}<.001$. Powel SN reported that axillary radiotherapy doubles the figures of lymphoedema against axillary surgery alone. Meek AG reported 2\% - 5\% of lymphoedema when radiation alone was given to the axilla.

There has always been a deep concern about cardiotoxicity in left-sided chest wall radiotherapy. Another important factor in this regard is the administration of anthracyclines. Radiotherapy following mastectomy has been seen to increase the risk of death due to cardiac reasons at almost 10 - 15 years after treatment.

The Swedish cancer registry data showed an increased risk of death due to myocardial infarction for patients with left-sided lesions (RR 1.09).
In our study there was drop only between $2 \%$ to $5 \%$ predominantly in the left-sided breast cancer patients, which was also seen in conventional arm, again statistically proving to be insignificant. However, cardiac toxicities are a late manifestation and long-term follow-up is required.(11)

\section{CONCLUSION}

The treatment schedule and the technique are feasible to deliver without affecting the quality of life of the patient. All the toxicity parameters are within acceptable limits. Hypofractionation can be easily followed in Institutions with high work load due to equal outcome.

\section{REFERENCES}

[1] Julka PK. Breast cancer cases in India to double 2015. All India institute of Medical Sciences (AIIMS) 2011.

[2] Edge SB, Byrd DR, Compton CC, et al. American Joint Committee on Cancer (AJCC). $7^{\text {th }}$ edn. New York: Springer 2010.

[3] Pierce LJ. The use of radiotherapy after mastectomy: a review of the literature. J Clin Oncol 2005;23(8):170617.

[4] Plataniotis G. Hypofractionated radiotherapy in the treatment of early breast cancer. World Journal of Radiology 2010;2(6):197-202.

[5] Whelan TJ, Jean-Philippe P, Levine MN, et al. LongTerm Results of Hypofractionated Radiation Therapy for Breast Cancer. The New Eng J Med 2010;362:51320.

[6] START Trialists' Group, Bentzen SM, Agrawal RK, et al. The UK Standardisation of Breast Radiotherapy (START) Trial A of radiotherapy hypofractionation for treatment of early breast cancer: a randomised trial. Lancet Oncol 2008;9(4):331-41.

[7] START Trialists' Group, Bentzen SM, Agrawal RK, et al. The UK Standardisation of Breast Radiotherapy (START) Trial B of radiotherapy hypofractionation for treatment of early breast cancer: a randomised trial. Lancet 2008;371(9618):1098-107.

[8] Brenner DJ. The linear-quadratic model is an appropriate methodology for determining isoeffective doses at large doses per fraction. Semin Radiat Oncol 2008;18(4):234-9.

[9] Jones B, Dale RG, Deehan C, et al. The role of Biologically Effective Dose (BED) in clinical oncology. Clin Oncology (R Coll Radiol) 2001;13(2):71-81.

[10] Shahid A, Athar MA, Asghar S, et al. Post mastectomy adjuvant radiotherapy in breast cancer: a comparision of three hypofractionated protocols. J Pak Med Assoc 2009;59(5):282-7.

[11] Jagsi R, Griffith KA, Koelling $T$, et al. Rates of Myocardial Infarction and coronary artery disease and risk factors in patients treated with radiation therapy for early-stage breast cancer. Cancer 2007;109(4):650-7. 